\title{
Efficacy of Combined Antithrombotic, Statins and Anti-Hypertensive Drugs in Acute Ischemic Stroke
}

\author{
Ramesh Sharma Poudel, ${ }^{1}$ Lekhjung Thapa, ${ }^{2}$ Shakti Shrestha, ${ }^{3}$ Dipendra Khatiwada, ${ }^{4}$ Nijan Upadhyay, ${ }^{1}$ Tirtha \\ Raj Bhandari, ${ }^{5}$ Asis Shrestha ${ }^{6}$ \\ 'Department of Pharmacy, National Model College for Advance Learning, Kathmandu, Nepal, ${ }^{2}$ Department of Neurology, \\ College of Medical Sciences Teaching Hospital, Chitwan, Nepal, ${ }^{3}$ Department of Pharmacy, Shree Medical and Technical \\ College, Chitwan, Nepal, ${ }^{4}$ Department of Community Medicine, College of Medical Sciences Teaching Hospital, Chitwan, \\ Nepal, 5 Jutpani Primary Health Centre, Chitwan, Nepal, ${ }^{6}$ Khairenitar Primary Health Centre, Nuwakot, Nepal.
}

Introduction: Anti-thrombotic, statins and antihypertensive drugs are commonly used for the management of acute ischemic stroke in Nepal but there is no evidence regarding their efficacy in our context. This study aims to explore the efficacy of these combined medications (anti-thrombotic, statins and antihypertensive drugs) in acute ischemic stroke patients for the first time and to assess the risk factors present in those patients.

Methods: The study was conducted on 37 acute ischemic stroke patients admitted and treated at the Neurology Department of College of Medical Sciences-Teaching Hospital, Chitwan, Nepal and they were followed at three months ( $90 \pm 10$ days). The initial stroke severity and functional disability were evaluated at admission and at 3 months using National Institute of Health Stroke Scale (NIHSS) and Modified Rankin Scale (mRS) respectively. The outcome parameters were functional independence $(m R S \leq 2)$ and mortality at three months $(m R S=6)$. Frequency distribution, Mann-Whitney U-test and $\chi^{2}$ test were performed for statistical analysis.

Results: Out of 37 patients, 27 (73\%) were Smoker, 22 (59.5\%) had hypertension and 19 (51.4\%) were alcohol consumer. The stroke severity on admission was statistically significant with functional independence $(\mathrm{P}=0.003)$ and mortality $(\mathrm{P}=0.015)$ at three month. Similarly, Rankin grade on admission was statistically significant with functional independence $(\mathrm{P}=0.001)$ and mortality $(\mathrm{P}=0.048)$ at three month. At three months, 20 (69\%) survived patients were independent while mortality was seen in eight $(21.6 \%)$ patients.

Conclusions: The management of acute ischemic stroke with combined anti-thrombotic, statins and antihypertensive drugs seems promising and efficacious in mild to moderately severe ischemic stroke.

Keywords: antihypertensive; anti-thrombotic; efficacy; statins; stroke.

\section{INTRODUCTION}

Stroke is a second leading cause of death worldwide. ${ }^{1}$ Among stroke, ischemic stroke is the most common. ${ }^{2}$ In 2005, 16 million people were afflicted with first ever stroke and 5.7 million died because of the effects of stroke. ${ }^{3}$ Reliable statistics about the stroke are not available from Asia. ${ }^{4}$ However, burden of stroke in Asia is forecasted to increase. ${ }^{5}$ In Nepal nearly 50,000

Correspondence: Ramesh Sharma Poudel, Hospital Pharmacy Chitwan Medical College Teaching Hospital, Chitwan, Nepal. Email: pharmacistsharma23@gmail.com, Phone:+977-9843089582. 
Poudel et al. Efficacy of Combined Antithrombotic, Statin and Anti-Hypertensive drugs in Acute Ischemic Stroke.

people are afflicted by stroke every year, with 15,000 annual deaths. ${ }^{6}$ Intravenous Alteplase is currently the only FDA-approved therapy for treatment of patients with acute ischemic stroke and its use is associated with the improved outcomes. ${ }^{7}$ Unfortunately, Alteplase is very rarely used in limited number of hospitals of Nepal due to its high cost and patients often arrive at the hospital beyond the standard time required for the treatment. Combined medications strategy, namely anti-thrombotic (antiplatelets/anticoagulants), statin with antihypertensive drugs are commonly used in the management of acute ischemic stroke. But, there is no evidence regarding their efficacy in our context. This study aimed to determine the efficacy of these combined medications for acute ischemic stroke for the first time and to assess the risk factors present in those patients.

\section{METHODS}

A hospital-based prospective cross sectional study was performed on efficacy of combined medications, namely anti-thrombotic, statin and antihypertensive drugs in patients with acute ischemic stroke at Neurology Department of College of Medical Science-Teaching Hospital (CMS-TH), Chitwan, Nepal. The study was approved by the ethical committee of the institution, and a written informed consent was obtained from each patient or their relatives prior to the study. Acute ischemic stroke patient who met the inclusion criteria were included in the study from 25 May 2013 to 25 July 2013 and the same patients were followed at three months $(90 \pm 10$ days). Inclusion criteria includes acute ischemic age $>18$ years, modified Rankin Scale $(\mathrm{mRS})>2$, National Institute of Health Stroke Scale (NIHSS) $>0$ and should have received at least one dose of anti-thrombotic and statins. The detailed history, neurological examination including stroke severity and functional disability and radiological information were obtained using semi-structured questionnaire. NIHSS was used to accesses the stroke severity which had four categories namely normal $(\mathrm{NIHSS}=0)$, mild (NIHSS $=1-7)$, moderate (NIHSS $=7-14$ ) and severe (NIHSS $\geq 15$ ) while $\mathrm{mRS}$ was used to accesses the functional disability. All the patients were given antithrombotic drug, statin while antihypertensive drug (s) was given only for hypertensive patient on the basis of clinical judgements. Any supportive management was provided depending on the condition of the patients. Existence of stroke risk factors that included previous stroke, Coronary artery disease, Hyperlipidemia, Hypertension, Diabetes, Atrial fibrillation, Smoking, and alcohol consumption were noted. On the basis of efficacy, the outcomes were measured at three months. The modified Rankin score at three months was used to generate the two binary outcome variables namely 'death $(\mathrm{mRS}=6)$ ' and independence $(\mathrm{mRS}=0-2)$. The functional independency at three months accounted the percentage of survived patient only.

Mann-Whitney U-test was used to compare the medians and chi-square test was used to assess the association at $95 \% \mathrm{Cl}$. There were few missing data due to the death of the patient in which case valid percentage was calculated assigning the total number in that variable with ' $\mathrm{N}$ '.

\section{RESULTS}

Out of 37 patients, 27 (73\%) were males. The age ranged from 32-90 years with mean age of $63.43 \pm 16.34$. Thirty one $(83.3 \%)$ patients had first ever stroke. With respect to risk factors, 27 (73\%) were smoker, followed by $22(59.5 \%)$ had hypertension and $19(51.4 \%)$ were alcohol consumer. On admission, nine $(24.3 \%)$ patients had moderate disability $(m R S=3), 21$ $(56.8 \%)$ had moderately severe disability ( $m R S=4)$ and seven $(18.9 \%)$ had severe disability $(m R S=5)$. The degree of severity was mild (NIHSS $=1-7$ ) in $10(27 \%)$ patients, moderate (NIHSS $=8-14)$ in $5(13.5 \%)$ patients and severe (NIHSS $\geq 15)$ in $22(59.5 \%)$ patients. The onset to first dose antithrombotic time ranged from 3-64 hours. All the patients received Atorvastatin and antithrombotic drug while18 $(48.6 \%)$ received antihypertensive drug(s). At three months, 29(78.4\%) patients were survived and among them eight $(27.6 \%)$ patients had no stroke severity $(\mathrm{NIHSS}=0)($ Table 1$)$. Out of those survived (29), 20 (69\%) were functionally independent at three months (Table 2). 
Poudel et al. Efficacy of Combined Antithrombotic, Statin and Anti-Hypertensive drugs in Acute Ischemic Stroke.

\begin{tabular}{|c|c|c|}
\hline Characteristics $(\mathrm{N}=37)$ & Categories & n (\%) \\
\hline Age (years) & 32 to $90($ Mean $=63.43 \pm 16.34)$ & 37 (100) \\
\hline \multirow{2}{*}{ Gender } & Male & $27(73.0)$ \\
\hline & Female & $10(27.0)$ \\
\hline \multirow{2}{*}{ Previous stroke } & Yes & $6(16.2)$ \\
\hline & No & $31(83.8)$ \\
\hline \multirow{2}{*}{ CAD } & Yes & $1(2.7)$ \\
\hline & No & $36(97.3)$ \\
\hline Hyperlipidemia & No & $37(100)$ \\
\hline \multirow{2}{*}{ Hypertension } & Yes & $22(59.5)$ \\
\hline & No & $15(40.5)$ \\
\hline \multirow{2}{*}{ Diabetes } & Yes & $5(13.5)$ \\
\hline & No & $32(86.5)$ \\
\hline \multirow{2}{*}{ Atrial fibrillation } & Yes & $4(10.8)$ \\
\hline & No & $33(89.2)$ \\
\hline \multirow{2}{*}{ Smoking } & Yes & $27(73.0)$ \\
\hline & No & $10(27.0)$ \\
\hline \multirow{2}{*}{ Alcohol consumption } & Yes & $19(51.4)$ \\
\hline & No & $18(48.6)$ \\
\hline \multirow{3}{*}{ Rankin grade on admission } & Moderate disability (mRS 3) & $9(24.3)$ \\
\hline & Moderate severe disability (mRS 4) & $21(56.8)$ \\
\hline & Severe disability (mRS 5 ) & 7 (18.9) \\
\hline \multirow{3}{*}{ Stroke severity on admission } & NIHSS 1-7 (Mild) & $10(27.0)$ \\
\hline & NIHSS 8-14 (Moderate) & $5(13.5)$ \\
\hline & NIHSS $\geq 15$ (Severe) & $22(59.5)$ \\
\hline \multirow{2}{*}{ CT scan } & Yes & $36(97.3)$ \\
\hline & No & $1(2.7)$ \\
\hline \multirow{2}{*}{ MRI } & Yes & $7(18.9)$ \\
\hline & No & $30(81.1)$ \\
\hline Onset to first dose thrombolytic drug time (hrs) & 3 to $64 \mathrm{hrs}$ & $37(100)$ \\
\hline Door to scan time (hrs) & 0.5 to $14 \mathrm{hrs}$ & $37(100)$ \\
\hline Scan to first dose thrombolytic drug time (hrs) & 0.3 to $117 \mathrm{hrs}$ & $37(100)$ \\
\hline \multirow{2}{*}{ Thrombolytic drugs } & Aspirin & 34 (91.9) \\
\hline & Warfarin & $3(8.1)$ \\
\hline HMG-CoA reductase inhibitors & Atorvastatin & $37(100)$ \\
\hline \multirow{2}{*}{ Antihypertensive drug } & Yes & $18(48.6)$ \\
\hline & No & $19(51.4)$ \\
\hline \multirow{6}{*}{ Rankin grade at 3- months } & No symptoms at all (mRS 0) & $4(10.8)$ \\
\hline & No significant disability (mRS 1) & $9(24.3)$ \\
\hline & Slight disability (mRS 2) & 7 (18.9) \\
\hline & Moderate disability (mRS 3) & $5(13.5)$ \\
\hline & Moderate severe disability (mRS 4) & $4(10.8)$ \\
\hline & Death (mRS 6) & $8(21.6)$ \\
\hline \multirow{4}{*}{ Stroke severity at 3- months $(\mathrm{N}=29)$} & NIHSS 0 (Normal) & $8(27.6)$ \\
\hline & NIHSS 1-7 (Mild) & 11 (37.9) \\
\hline & NIHSS 8-14 (Moderate) & $8(27.6)$ \\
\hline & NIHSS $\geq 15$ (Severe) & $2(6.9)$ \\
\hline
\end{tabular}

7 JNMA | VDL 53 | N口. 1 || ISSUE 197 | JAN-MAR, 2015 
Poudel et al. Efficacy of Combined Antithrombotic, Statin and Anti-Hypertensive drugs in Acute Ischemic Stroke.

\begin{tabular}{|lll|}
\hline \multicolumn{2}{l}{ Table 2. Outcome Variables } \\
\hline Characteristics & Categories & $\mathbf{n}(\%)$ \\
Death at three months & Yes & $8(21.6)$ \\
& No & $29(78.4)$ \\
Independence at three & Yes & $20(69.0)$ \\
months & No & 9 (31.0) \\
\hline
\end{tabular}

The Mann-Whitney U-test (Table 3) showed that there is no significant association of outcomes variables at 3-months with age and onset to first dose of antithrombotic time (Table 3).
Chi-square test showed that death at three months was significantly associated with Rankin grade $(P=0.048)$ and stroke severity on admission $(P=0.015)$. Similarly, independency at three months was also significantly associated with Rankin grade $(\mathrm{P}=0.001)$ and stroke severity on admission $(P=0.003)$. Though independency at three months showed trend towards association with gender, previous stroke and atrial fibrillation, however, the associations was not statistically significant (Table 4).

Table 3. Comparison of median with outcome variables at 3-months.

Outcome ( $N=37)$

Characteristics

Death $(\mathrm{mRS}=6)$ at three months

Age (years)

Yes $(\mathbf{N}=8)$

No $(\mathbf{N}=29)$

P-value

Independent (mRS 0-2) at three months

Onset to first dose

69.5

67.0

0.651

Yes $(\mathbf{N}=20)$

No $(N=18)$

P-value

19.9

15.7

0.335

58.0

70.0

0.171

antithrombotic time (hrs)

19.9 .7

18.5

0.660

Table 4. Cross tabulation between categorical variables and outcome variables at three months.

\section{Outcome ( $N=37)$}

Characteristics

Death $(\mathrm{mRS}=6)$ at three months

Gender (Female)
Previous stroke
CAD
Hypertension

Diabetes

Atrial fibrillation

Smoking

Alcohol

Rankin grade on admission

Slight disability

Moderate disability

Moderately severe disability

Severe disability

Stroke severity on admission

NIHSS 1-7 (Mild)

Yes-n(\%)

4 (50.0)

No-n(\%)

0.0

6 (20.7)

P-value

6 (20.7)

0.174

0.0

1 (3.4)

0.305

5 (62.5)

$17(58.6)$

1.000

2 (25.0)

3 (10.3)

1.000

0.292

0.0

4 (13.8)

0.557

5 (62.5)

22 (75.9)

0.655

$15(51.7)$

1.000

$0.048 *$

$0.0 \quad 4(13.8)$

0.0

$5(17.2)$

5 (62.5)

$16(55.2)$

3 (37.5)

$4(13.8)$

$0.0 \quad 10(34.5)$

$0.015^{*}$

$5(17.2)$

0.0

$8(100.0)$

$14(48.3)$
Independent (mRS 0-2) at three months

Yes-n(\%) No-n(\%) P-value

$2(10.0) \quad 4(44.4) \quad 0.056$

$2(10.0) \quad 4(44.4) \quad 0.056$

$0 \quad 1(11.1) \quad 0.310$

$12(60.0) \quad 5(55.6) \quad 1.000$

$2(10.0) \quad 1(11.1) \quad 1.000$

$1(5.0) \quad 3(33.3) \quad 0.076$

$17(85.0) \quad 5(55.6) \quad 0.158$

$12(60.0) \quad 3(33.3) \quad 0.245$

0.001 *

$\begin{array}{ll}1(5.0) & 0 \\ 8(40.0) & 0 \\ 11(55.0) & 5(55.6) \\ 0 & 4(44.4)\end{array}$

$0.003 *$

$10(50.0) \quad 0$

$4(20.0) \quad 1(11.1)$

$6(30.0) \quad 8(88.9)$

\section{DISCUSSION}

In this study, 37 patients with a diagnosis of ischemic stroke were evaluated. Our study showed that stroke affected higher number of males than females $(73 \%$ vs
$27 \%$ ). Similar results have been reported in previous studies done in Nepal. ${ }^{5,8,9}$ A systematic review on sex differences in stroke epidemiology depicts that genetic factor, higher blood pressure, ischemic heart disease, 
Poudel et al. Efficacy of Combined Antithrombotic, Statin and Anti-Hypertensive drugs in Acute Ischemic Stroke.

peripheral artery disease and greater cigarette smoking are responsible for higher prevalence of stroke in males than females. Moreover, ovarian estrogens may protect females from ischemic stroke, at least of the non-cardioembolic type. ${ }^{10}$ Our study showed that the functional improvement after treatment was inferior in females than males with borderline significance. A review on sex differences in stroke outcomes concluded that women have less favorable outcomes after stroke than men. ${ }^{11}$

The mean age of stroke patients in our study population was 63.43 years (SD 16.34, range $32-90$ ). Some of the stroke studies in Nepal have reported low mean ages such as 58.27 years, 61 years and $59.09 \pm 14.21 .8,9$, 12 The patients included in these studies were younger than in our study. Our finding of 63.43 years compares well with a study on risk factors at Manipal Teaching Hospital, Pokhara, Nepal (65.98 years), in which age range of patients (34-91 years) was comparable with our study. ${ }^{13}$

Smoking is strong risk factor for both ischemic and haemorrhagic strokes. ${ }^{14}$ It is an important cause of atherosclerosis. Longer duration and increased number of cigarettes smoking has been reported to be significantly associated with ischemic stroke.$^{14}$ In our study, $73 \%$ of the subjects had a history of smoking, indicating smoking as the most common modifiable risk factor. Most of the previous studies in Nepal have also suggested smoking as a strong risk factor for stroke. ${ }^{8,10}$ In contrast, one study reported hypertension as most common modifiable risk factor. ${ }^{13}$

About $59.5 \%$ of the subjects in our study had hypertension (includes hypertension in the past), which was the second most prevalent risk factor. A study on stroke prevention and treatment suggested that there is a strong association between elevated blood pressure and stroke. ${ }^{15}$ Stroke studies in Nepal have reported $61.2 \%$, $47.2 \%$ and $40 \%$ of the study population with stroke had hypertension. 5,9,8 This suggests that hypertension is also a major risk factor of ischemic stroke in Nepalese population and treatment of hypertension is important and might be effective in stroke prevention.

Strong evidence indicates alcoholism and heavy drinking as being strong risk factor for all stroke subtypes. Majority of studies have evoked a protective effect in light or moderate drinkers and an increase risk in heavy drinkers. Increased level of HDL cholesterol, reduce platelet aggregation, and lower plasma fibrinogen concentration is observed in lightto-moderate drinkers while heavy alcohol consumption can lead to hypertension, hypercoagulability, reduced cerebral blood flow, and a greater likelihood of atrial fibrillation. ${ }^{16}$ Our study showed that $51.4 \%$ patients consumed alcohol. It is comparable with several studies done in Nepal. ${ }^{5,8,13}$ The practice of alcohol consumption in Nepalese community also makes it a meaningful risk factor.

Previous stroke as a risk factor was present in $16.2 \%$ of the cases. None of the studies done in Nepal have reported it. However, one study in Germany has shown previous stroke as one of the risk factors. ${ }^{17}$ Our analysis showed borderline association between independence on $90 \pm 10$ days and previous stroke $(P=0.056)$.

Diabetes mellitus was present in $13.5 \%$ of the cases. This result is comparable with the other studies done in Nepal. $5,8,9,13$ This suggests that diabetes mellitus as a risk factor for stroke appears to be less prominent in Nepalese population.

Atrial fibrillation was present in $10.8 \%$ of the cases. It is comparable with a retrospective study of stroke and its risk factors at Nepal Medical College Teaching Hospital ${ }^{9}$ but a study of risk factors of stroke in patients admitted in Manipal Teaching Hospital, Pokhara, reported about $24 \%$ of patients with atrial fibrillation. ${ }^{13}$

None of the patients in our study had a history of hyperlipidemia. But studies have shown a clear relationship between dyslipidemia and the risk of ischemic stroke in both men and women. The Asia Pacific Cohort Studies Collaboration $(n=352033)$ found a $25 \%$ increase in ischemic stroke rates for every $1-\mathrm{mmol} / \mathrm{L}$ (38.7-mg/dL) increase in total cholesterol. ${ }^{16}$ Retrospective analysis of stroke and its risk factors at Bir Hospital, Nepal reported hyperlipidemia in $9.05 \%$ of the cases. ${ }^{5}$ Their role as risk factor of stroke might need further elaboration in larger population.

The Coronary artery disease was reported in $2.7 \%$ of the cases in this study. None of the studies previous conducted in Nepal have mentioned CAD as risk factor, however, a clinico-radiological profile of stroke in eastern Nepal reported heart disease as risk factor in $6 \%$ of patients. ${ }^{8}$

Several scales have been developed to quantify neurological impairments following ischemic stroke, and scores can be used to predict recovery from stroke. The National Institutes of Health Stroke Scale is a widely used rating instrument to measure neurological deficits. ${ }^{18}$ Initial stroke severity is the only significant, independent, and modifiable risk factor for Neurologic deterioration (ND). ${ }^{19}$ In this study all patients had some degree of stroke severity with severe stroke in most patients $(59.5 \%)$ on admission. The severity of stroke markedly reduced after treatment at three months. About $27.6 \%$ patients became normal and the percentage of severe stroke reduced by almost $53 \%$. This shows a 
Poudel et al. Efficacy of Combined Antithrombotic, Statin and Anti-Hypertensive drugs in Acute Ischemic Stroke.

promising result of the treatment. The stroke severity on admission is significantly associated with both death $(\mathrm{P}=0.015)$ and independence $(\mathrm{P}=0.003)$. About $21.6 \%$ of total patients who had severe stroke died and almost same percentage of patients with similar severity were dependent at the end of the study. A study on stroke mortality in the Intensive Care Unit of same hospital depicted $19.05 \%$ death within seven days. ${ }^{20}$ Patients with less severe stroke were found to recover more rapidly after the treatment than more severe ones suggesting the necessity of an additional intervention in severe patients. However, the cause of ischemic stroke might also influence the recovery. The TOAST study showed that two third of the study population on admission had NIHSS $\leq 3$ and achieved an excellent outcome on seven days, but very few patients with a baseline NIHSS $>15$ achieved excellent results on 90 days. It also depicts that patients with severe impairments (NIHSS score $>15$ ) have less than a $20 \%$ chance of achieving an excellent outcome. ${ }^{18}$

The entire admitted patient had some degree of functional dependency based on mRS (24.3\% moderate, $56.8 \%$ moderately severe and $18.9 \%$ severe). After the treatment, out of 29 alive patients $69 \%$ were functionally independence (mRS $\leq 2)$ and the Rankin grade on admission was statistically significant with both death $(P=0.048)$ and functional independency $(P=0.002)$. This association signifies higher number of death and functional dependency at three months in patients with higher functional disability. This study also indicates that combined medications are less effective in patient with higher degree of disability. However, the relationship between functional admission scores and improvement in function is less clear. ${ }^{21}$ Moreover, infarct subtype and risk factors also influence the outcomes of therapy. A prospective study of consecutive patients with acute stroke showed nonmodifiable risk factor such as young age group (5675 years) was protective factors while modifiable risk factors such as atrial fibrillation, ischemic heart disease, diabetes, and ex-smoker status were risk factors for early death in patient with acute ischemic stroke. ${ }^{22}$
Patient treated with combined medications namely antithrombotic, statin and antihypertensive drug showed functional improvement (69\%) at three months in our study. Study reported oral anticoagulants or statins used in hospital were associated with better stroke outcome. ${ }^{23}$ Similarly, a study on potential effects of statins initiated before or within four weeks of stroke demonstrated that post stroke statins were associated with a significant probability of a favorable outcome at 90 days. ${ }^{24}$ However, the Chinese Acute Stroke Trial showed that the rates of long-term complete recovery or death and disability were not significantly improved in patients treated with aspirin. ${ }^{25}$

This study limits to small study population, unclassified stroke subtype, use of more than one antihypertensive drugs and inability to compare the outcomes with untreated patients.

\section{CONCLUSIONS}

The management of acute ischemic stroke with combined medications (antithrombotic, statins and antihypertensive) seems promising. However, this study needs further elaboration on larger population to support itself. Combined medications namely antithrombotic, statin and antihypertensive drugs show good functional improvement in patient with less severe stroke, regardless of stroke subtypes. The establishment of 'Comprehensive Stroke Units' and' Stroke Registry' might be necessary for better care of patient and future research.

\section{ACKNOWLEDGEIMENTS}

We would like to thank Prof. Dr. PVS Rana, Dr. Baburam Pokhrel, Dr. Ghan shyam Kheral, Dr. Prakash Bhattari, Bipen Shah, Naresh Manandhar, Pawan Bhatta, participants and their relatives and nursing staff of Neurology Department at College of Medical Sciences Teaching Hospital for their assistance, support and guidance during data collection, analysis and writing.

\section{REFERENCES}

1. Lopez AD, Mathers CD, Ezzati M, Jamison DT, Murray CJ. Global and regional burden of disease and risk factors, 2001: systematic analysis of population health data. Lancet. 2006;367(9524):1747-57.

2. American Heart Association, 2012. Let's talk about ischemic stroke and their causes [online]. (cited 2014 Jan 06) Available from: URL:http://www.strokeassociation.org/idc/groups/ stroke-public/@wcm/@hcm/documents/downloadable/ ucm_309725.pdf.
3. Strong K, Mathers C, Bonita R. Preventing stroke: saving lives around the world. Lancet Neurol. 2007;6(2):182-7.

4. Singh RB, Suh IL, Sing VP, Chaithiraphana S, Latothavorn P, Sy RG, et al. Hypertension and stroke in Asia: Prevalence, control and strategies in developing countries for prevention. J Hum Hypertens. 2000;14(10-11):749-63. 
Poudel et al. Efficacy of Combined Antithrombotic, Statin and Anti-Hypertensive drugs in Acute Ischemic Stroke.

5. Shrestha A, Shah DB, Adhakari KR, Sapkota S, Regmi PR. Retrospective Analysis of Stroke and Its Risk Factors at Bir Hospital. PMJN. 2011;11(2):28-30

6. Shaik MM, Loo KW, Gan SH. Burden of stroke in Nepal. Int J Stroke. 2012;7(6):517-20.

7. Adams HP Jr, del Zoppo G, Alberts MJ, Bhatt DL, Brass L, Furlan A, et al. Guidelines for the Early Management of Adults With Ischemic Stroke: A Guideline From the American Heart Association/American Stroke Association Stroke Council, Clinical Cardiology Council, Cardiovascular Radiology and Intervention Council, and the Atherosclerotic Peripheral Vascular Disease and Quality of Care Outcomes in Research Interdisciplinary Working Groups: The American Academy of Neurology affirms the value of this guideline as an educational tool for neurologists. Circulation. 2007; 115(20):e478-534.

8. Naik M, Rauniyar RK, Sharma UK, Dwivedi S, Karki DB, Samuel JR. Clinico-radiological profile of stroke in eastern Nepal: a computed tomographic study. Kathmandu Uni Med J. 2006;4(2):161-66.

9. Devkota KC, Thapamagar SB, Malla S. Retrospective analysis of stroke and its risk factors at Nepal Medical College Teaching Hospital. Nepal Med Coll J. 2006;8(4):269-75.

10. Appelros P, Stegmayr B, Terent A. Sex differences in stroke epidemiology: a systematic review. Stroke. 2009;40(4):1082-90.

11. Reeves MJ, Bushnell CD, Howard G, Gargano JW, Duncan PW, Lynch G, et al. Sex differences in stroke: epidemiology, clinical presentation, medical care, and outcomes. Lancet Neurol. 2008;7(10):915-26.

12. Pathak V, Kanth R, Pant H. Stroke: a Case series study in Nepal Medical College Teachin Hospital. Nepal Med Coll J. 2006;8(3):180-1

13. Maskey A, Parajuli M, Kholi SC. A Study of Risk Factors of Stroke in Patients Admitted in Manipal Teaching Hospital, Pokhara. Kathmandu Uni Med J. 2011;9(36):244-7.

14. Uddin MJ, Mondol BA, Ahmed S, Ullah AA, Jabbar M, Mohammad QD. Smoking and ischemic stroke. Bangladesh Journal of Neuroscience. 2008;24(1):50-54.

15. Marsh JD, Keyrouz SG. Stroke prevention and treatment. J Am Coll Cardiol. 2010;56(9):683-91.

16. Goldstein LB, Adams R, Alberts MJ, Appel LJ, Brass LM, Bushnell CD, et al. Primary Prevention of Ischemic Stroke: a Guideline from the American Heart Association/American Stroke Association Stroke Council: cosponsored by the Atherosclerotic Peripheral Vascular Disease Interdisciplinary Working Group; Cardiovascular Nursing Council; Clinical Cardiology Council; and the Quality of Care and Outcomes Research Interdisciplinary Working Group: the American Academy of Neurology affirms the value of this guideline. Stroke. 2006;37(6):1583-633.
17. Grau AJ, Weimar C, Buggle F, Heinrich A, Goertler M, Neumaier S, et al. Risk factors, outcome, and treatment in subtypes of ischemic stroke: the German stroke data bank. Stroke. 2001;32(11):2559-66.

18. Adams HP Jr, Davis PH, Leira EC, Chang KC, Bendixen $\mathrm{BH}$, Clarke WR, et al. Baseline NIH stroke scale score strongly predicts outcome after stroke a report of the trial of org 10172 in acute stroke treatment (TOAST). Neurology.1999;53(1):126-31

19. Siegler JE, Boehme AK, kumar AD, Gillette MA, Albright KC, Beasley TM, Martin-Schild S. Identification of modifiable and nonmodifiable risk factors for neurologic deterioration after acute ischemic stroke. J Stroke Cerebrovasc Dis. 2013;22(7):e207-13.

20. Thapa L, Shrestha A, Pokhrel B, Paudel R, Rana PVS. Stroke mortalty in intensive care unit from tertiary care neurological centre. J Nepal Med Assoc. 2013;52(190):332-6.

21. Jongbloed L. Prediction of function after stroke: a critical review. Stroke. 1986;17(4):765-76.

22. Wong K. Risk Factors for Early Death in Acute Ischemic Stroke and Intracerebral Hemorrhage: A Prospective Hospital-Based Study in Asia. Asian Acute Stroke Advisory panel. Stroke. 1999;30(11):2326-30.

23. Niewada M, Sarzynska-Dlugosz IM, Skowronska M, Kaminski B, Kobayashi A, Czlonkowska A. Pharmacotherapy prior to and in acute ischaemic stroke. The use of pharmacotherapy and drug-associated outcomes in real world practice - findings from the Polish Hospital Stroke Registry. Neurol Neurochir Pol. 2013;47(6):509-16.

24. Moonis M, Kane K, Schwiderski U, Sandage BW, Fisher M. HMG-CoA reductase inhibitors improve acute ischemic stroke outcome. Stroke. 2005;36(6):1298-300.

25. CAST (Chinese Acute Stroke Trial) Collaborative Group CAST:radomised placebo-controlled trial of early aspirin use in 20,000 patients with acute ischaemic stroke. Lancet. 1997;349(9066):1641-49. 